\title{
Biological application of multi-component nanowires in hybrid devices powered by $\mathbf{F}_{1}$-ATPase motors
}

\author{
Quan Ren · Ya-Pu Zhao $\cdot$ J. C. Yue · Y. B. Cui
}

Published online: 20 May 2006

C Springer Science + Business Media, LLC 2006

\begin{abstract}
In this paper, construction of hybrid device by integrating nanowires with $\mathrm{F}_{1}$-ATPase motors is described. The nickel nanowires and multi-segment nanowires, including gold and nickel, were fabricated by electrochemical deposition in nanoporous templates. The nickel nanowires functionalized by biotinylated peptide can be assembled directly onto $\mathrm{F}_{1}$-ATPase motors to act as the propellers. If the multicomponent nanowires, including gold and nickel, were selectively functionalized by the thiol group modified ssDNA and the synthetic peptide, respectively, the biotinylated $\mathrm{F}_{1}$ ATPase motors can be attached to the biotinylated peptide on nickel segment of the nanowires. Then, the multi-component nanowires can also be used as the propellers, and one may observe the rotations of the multi-component nanowires driven by $\mathrm{F}_{1}$-ATPase motors. Therefore, introduction of multiple segments along the length of a nanowire can lead to a variety of multiple chemical functionalities, which can be selectively bound to cells and special biomolecules. This method provides an insight for the construction of other hybrid devices with its controlling arrangement of different biomolecule on designed nanometer scale structures.
\end{abstract}

Keywords Biomolecular motors $\cdot \mathrm{F}_{1}$-ATPase . Nanofabrication $\cdot$ Electrochemical deposition.

Q. Ren · Y.-P. Zhao $(\bowtie)$

State Key Laboratory of Nonlinear Mechanics (LNM), Institute of Mechanics, Chinese Academy of Sciences, Beijing 100080, China e-mail: yzhao@lnm.imech.ac.cn

J. C. Yue · Y. B. Cui

Institute of Biophysics, Chinese Academy of Sciences, Beijing 100101, China

\section{Introduction}

Constructing hybrid devices, based on molecular biology and micro/nano fabrication, is increasingly becoming a hot topic (Noji et al., 1997; Soong et al., 2000, 2001; Fritz et al., 2000; Huang et al., 2004; Jia et al., 2004; Ren and Zhao, 2006). These hybrid devices offer the potential application in near future, such as in the development of many types of advanced biosensors and force bioactuators used in the medical and therapeutic area. Of those hybrid systems, much attention has been paid to a wide range of motor proteins (Lee et al., 2003), such as myosin (Dobbie et al., 1998; Kitamura et al., 1999), kinesin (Block, 1998; Hunt et al., 1994), dynein (Gibbons, 1988; Shingyoji et al., 1998), flagellar (Berry and Armitage, 1999; Kaiser, 2000), and ATPase (Adenosine TriPhosphate synthase) (Noji et al., 1997; Soong et al., 2000; Yasuda et al., 1998; Montemagno and Bachand, 1999; Bachand and Montemagno, 2000), because they can convert the chemical energy derived from the ATP hydrolysis into mechanical work. Among them, myosin, dynein and kinesin are linear motors, which can carry a cargo and move along a fixed direction. While ATPase motors and flagellar motor are rotary motors, which can rotate in different directions. Of those rotary motors, ATPase motor, ubiquitous in organisms from bacteria to man, is the best characterized protein in terms of its atomic structure and biochemistry. It has similar structures, consisting of two rotary assemblies, $\mathrm{F}_{0}\left(\mathrm{ab}_{2} \mathrm{c}_{\mathrm{n}}\right)$ and $\mathrm{F}_{1}\left(\alpha_{3} \beta_{3} \gamma \delta \varepsilon\right)$ connected by a common elastic shaft, subunit $\gamma$. The mechanism of ATPase motor is related to the basic physical principle of the ATPase: Boyer's binding change principles for ATP synthesis on $\mathrm{F}_{1}$ (Boyer, 1993) and Cox's principle for transport proton in $\mathrm{F}_{0}$ (Cox et al., 1986). However, not until the first atomic-level $(0.28 \AA)$ resolution structure of bovine mitochondria $F_{1}$ was obtained (Abrahams et al., 1994), as well as a great deal of related 
Fig. 1 Schematic of $F_{1}$-ATPase motor, whose propeller is nickel nanowire fabricated by electrochemical deposition

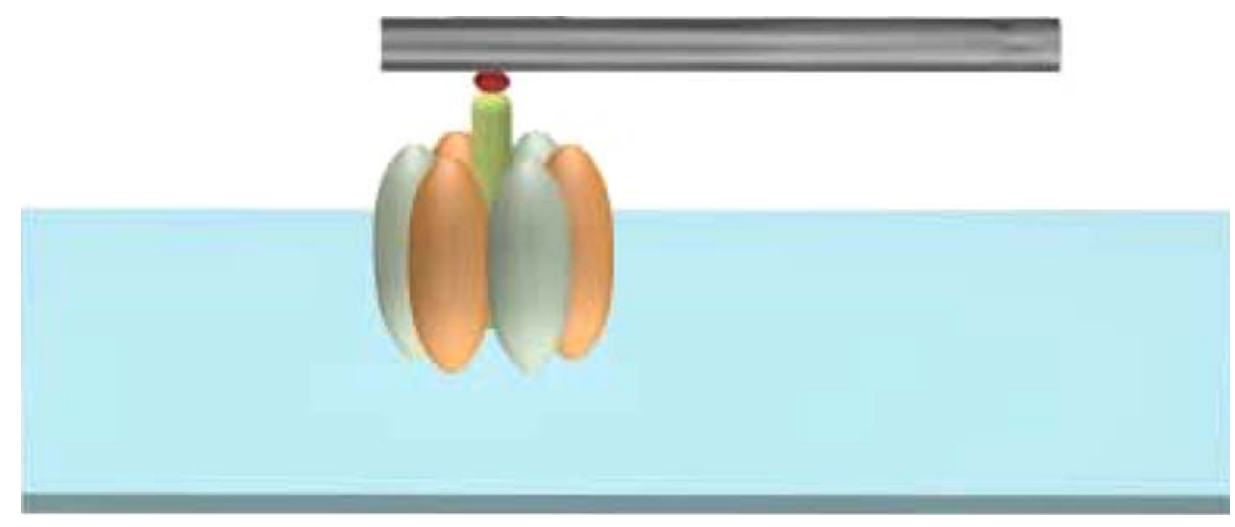

studies (Duncan et al., 1995; Ogilvie et al., 1997; Engelbrecht and Junge, 1997; Groth and Walker, 1997), the understanding of the mechanism of $F_{1}$ motor was turned from the 'cartoon' stage to detailed calculations (Noji et al., 1997; Oster and Wang, 2003). Now, the prevailing and accepted mechanism for isolated $F_{1}$-ATPase is that the central $\gamma$ subunit rotates against the surrounding $\alpha_{3} \beta_{3}$ subunits during the hydrolysis of ATP in the three catalytic $\beta$ subunits of the $\mathrm{F}_{1}$-ATPase. And the reverse rotation of $\gamma$ subunit in ATP synthase, powered by the proton flow, is supposed to result in the ATP synthesis in the three $\beta$ subunits. However, the experimental realization of the $F_{1}$-ATPase rotation is difficult. The initial direct and visual rotation is observed by using a fluorescent actin filament attached to $\gamma$ subunit (Yasuda et al., 1998), which provides the direct convincing evidence of the rotation of the $\mathrm{F}_{1}$-ATPase motor. In addition, other researchers have also observed the rotation of the $\mathrm{F}_{1}$ ATPase motor (Omote et al., 1999; Nishio et al., 2002; Pänke et al., 2000). Further, Noji and Yasuda used small colloidal gold bead of $40 \mathrm{~nm}$ in diameter as the propeller to study the mechanism of stepping of the $\mathrm{F}_{1}$-ATPase motor with highspeed imaging (Yasuda et al., 2001). All those studies show the possibility of application of $F_{1}$-ATPase motor. Later, the nanoscale biomolecule, $\mathrm{F}_{1}$-ATPase, was integrated with the inorganic NEMS to construct a hybrid device (Soong et al., 2000, 2001; Montemagno and Bachand, 1999; Bachand and Montemagno, 2000). In these works, the engineered propellers made in PMMA coated with nickel were fabricated and assembled on the motors. However, it is difficult to fabricate the nanoscale structures by NEMS technology. If the applications of $F_{1}$-ATPase motor were expanded in physical sciences, it requires the development of an easy method to fabricate and functionalize the propellers for the $F_{1}$-motors. The electrochemical deposition technique provides a possibility with its broad range of applications in nanowires (Reich et al., 2003; Salem et al., 2004; Lapoinet et al., 2004).

In this paper, nickel nanowires were fabricated by electrochemical deposition in nanoporous templates. After functionalized by the biotinylated peptide with a sequence of His-His-His-His-His-His-Gly-Asp-Gly-Cys, the nickel nanowires can be used as the propellers of the $F_{1}$-ATPase motor as shown schematically in Fig. 1. In addition, a multi-component nanowire with three segments $\mathrm{Ni} / \mathrm{Au} / \mathrm{Ni}$ was also fabricated by electrochemical deposition. And the thiol group modified ssDNA and the biotinylated peptide were designed to selectively bind to the gold and nickel segments, respectively, by which the $\mathrm{F}_{1}$-ATPase motor can only be attached to the nickel segment of the nanowires by biotin-streptavidin linkage. The final observation of rotation of propeller driven by motors demonstrates that the multi-component nanowires accompanied by the different biomolecules can be designed to construct the hybrid device.

\section{Materials and methods}

\subsection{Preparation of the $\mathrm{F}_{1}$-ATPase}

$\mathrm{F}_{1}$-ATPase, whose coding sequence was isolated from thermophilic bacterium, Bacillus PS3, was a kindly gift from Dr. Montemagno. Thermophilic bacterium, Bacillus PS3, with ten histidine on $\beta$ subunits were expressed and purified as follows (Matsui and Yoshida, 1995). The expressed strain was cultured in $2 \times \mathrm{YT}$ medium $\left(\mathrm{AMP}^{+}\right)$at $37^{\circ} \mathrm{C}$ for $4 \mathrm{~h}$. When the value of $A_{660}$ is between 0.6 and 0.8 , the expression of the $\mathrm{F}_{1}$-ATPase was induced by addition of $1 \mathrm{mmol} / \mathrm{L}$ isoprophylthio- $\beta$-D-galactoside for $3 \mathrm{~h}$. Then, the products were collected after centrifuged at $4000 \times \mathrm{g}$ for $15 \mathrm{~min}$. The resuspension of products at a $50 \mathrm{mmol} / \mathrm{L}$ Tris- $\mathrm{HCl}$ buffer set to $\mathrm{pH} 8.0$ were added into lysozyme of $1 \mathrm{mg} / \mathrm{mL}$ to react at $4{ }^{\circ} \mathrm{C}$ for $30 \mathrm{~min}$, followed by sonication in buffer $\mathrm{A}(50 \mathrm{mmol} / \mathrm{L}$ Tris- $\mathrm{HCl}$ of $\mathrm{pH} 8.0,0.5 \mathrm{~mol} / \mathrm{L}$ $\mathrm{NaCl}$ and $1 \mathrm{mmol} / \mathrm{L}$ phenylmethan sulphonyl fluoride). The extracts were incubated at $60^{\circ} \mathrm{C}$ for $30 \mathrm{~min}$, and purified by using $\mathrm{Ni}^{2+}$-NTA affinity chromatography at $4^{\circ} \mathrm{C}$. Finally, the products were biotinylated immediately after purification. 


\subsection{Nanowires synthesis and chemical functionalization}

The electrochemical deposition technique was used to fabricate the propellers of $F_{1}$-ATPase motors. The filter template for electrochemical deposition, in which nanowires can be synthesized, was formed by anodic oxidation of aluminum. First, a gold film was sputter-deposited on one side of the porous $\mathrm{Al}_{2} \mathrm{O}_{3}$ template to serve as a working electrode. Second, the nickel nanowires or the multi-component nanowires were electrochemically synthesized into nanometer wide pore of $\mathrm{Al}_{2} \mathrm{O}_{3}$ template of $15 \mathrm{~nm}$ in a nominal pore diameter. In our experiments, the nickel nanowires were deposited from a solution of $1 \mathrm{~mol} / \mathrm{L} \mathrm{NiCl} 2 \cdot 6 \mathrm{H}_{2} \mathrm{O}$ and $0.25 \mathrm{~mol} / \mathrm{L} \mathrm{H}_{3} \mathrm{BO}_{3}$ buffered to $\mathrm{pH} 3.4$ at a potential of $-0.7 \mathrm{~V}$. And the multicomponent nanowires, $\mathrm{Ni} / \mathrm{Au} / \mathrm{Ni}$, were deposited from a solution of $1 \mathrm{~mol} / \mathrm{L} \mathrm{NiCl}_{2} \cdot 6 \mathrm{H}_{2} \mathrm{O}, 0.05 \mathrm{~mol} / \mathrm{L} \mathrm{HAuCl} \mathrm{H}_{4} \cdot 2 \mathrm{H}_{2} \mathrm{O}$ and $0.25 \mathrm{~mol} / \mathrm{L} \mathrm{H}_{3} \mathrm{BO}_{3}$ buffered to $\mathrm{pH} 3.4$ at a potential of $-0.05 \mathrm{~V}$ for gold and $-0.7 \mathrm{~V}$ for nickel, respectively. The length of nanowires can be controlled within a range of 1$2 \mu \mathrm{m}$. The wires are therefore nanometers in diameter and microns in length. Before being attached to the motors, the nanowires must be functionalized by fluorescently labeled molecule for observation and attachment. There are several steps in the way of functionlization of the nickel nanowires as shown schematically in Fig. 2(a-1), (b-1) and (c-1). For the multi-component nanowires, $\mathrm{Ni} / \mathrm{Au} / \mathrm{Ni}$, one may selectively functionalize specific segments in them to direct the binding of $F_{1}$-ATPase motor to the specific regions of the wires. Figure 3 schematically shows our approach for synthesis and functionalization, which is similar to that for nickel nanowires. When the nanowires were deposited into the alumina filter template, the gold electrode on the template
Fig. 2 Schematic of preparation and functionalization of nickel nanowires. (a-1) Nanowires were fabricated by electrochemical deposition into alumina filter template, and the filter template was adhered on a silicon flake, (b-1) $\mathrm{Al}_{2} \mathrm{O}_{3}$ on the silicon was removed by $\mathrm{NaOH}$ solution, (c-1) functionalizing the nickel nanowire, and the corresponding products, (a-2) SEM picture of $\mathrm{Al}_{2} \mathrm{O}_{3}$ template, (b-2) TEM picture of nickel nanowires, (c-2) fluorescent image of nickel nanowires

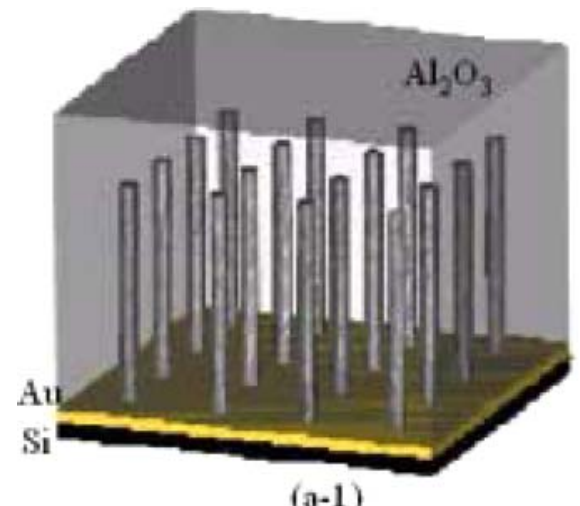

$(a-1)$

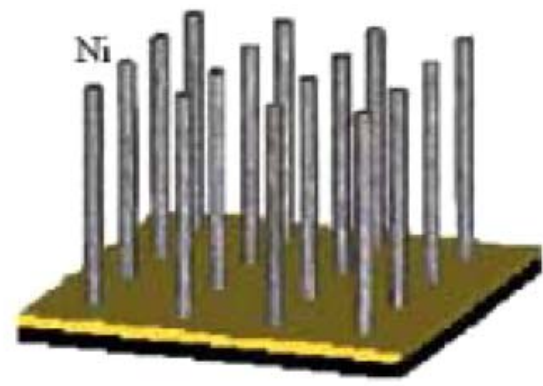

(b-1)

Flworescently

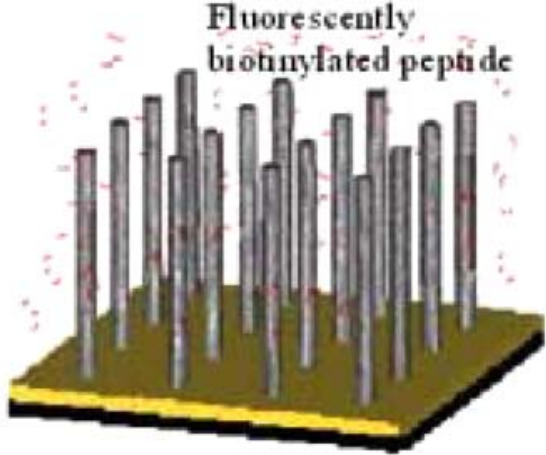

(c-1)

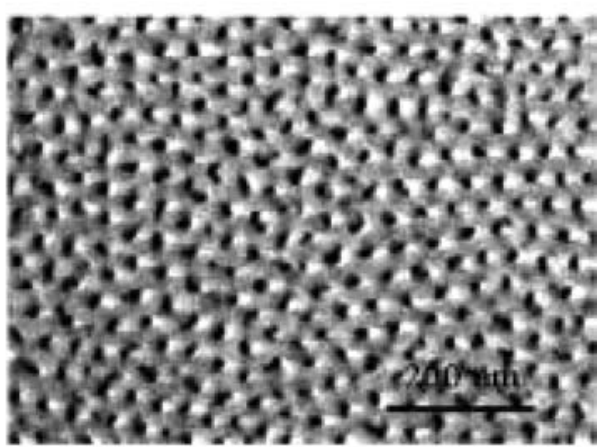

(3-2)

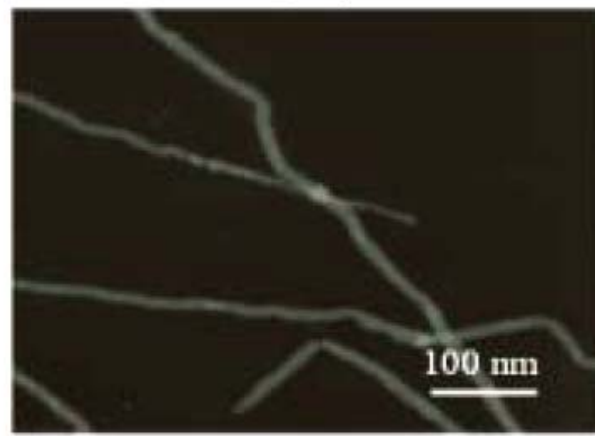

(b-2)

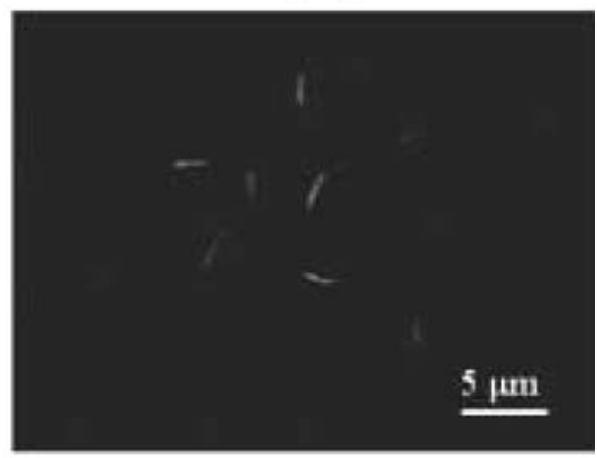

(c-2) 
Fig. 3 Schematic of preparation and functionalization of multi-component nanowires. (a) Multi-component nanowires were fabricated by the electrochemical deposition into alumina filter template, and the filter template was adhered on a silicon flake, (b) $\mathrm{Al}_{2} \mathrm{O}_{3}$ on the silicon was removed by $\mathrm{NaOH}$ solution, (c) thiol group modified ssDNA was used to coat on the gold segment on the multi-component nanowires, (d) Biotinylated pepetide labeled by fluorescent material, FITC, was used to functionalize the nickel segment on the multi-component nanowires

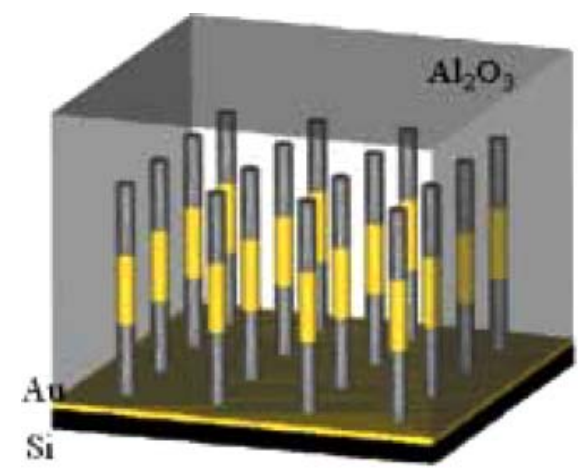

(a)

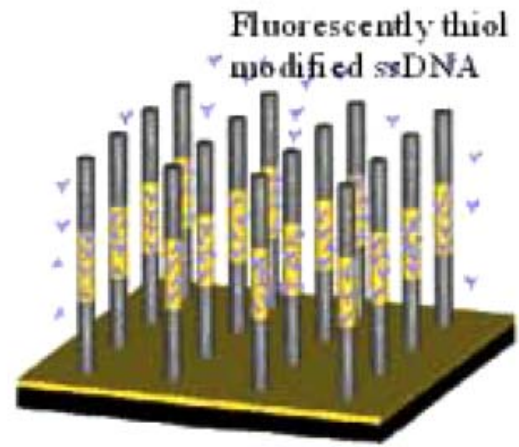

(c)

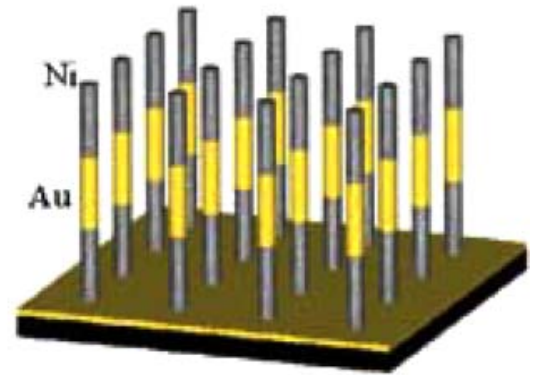

(b)

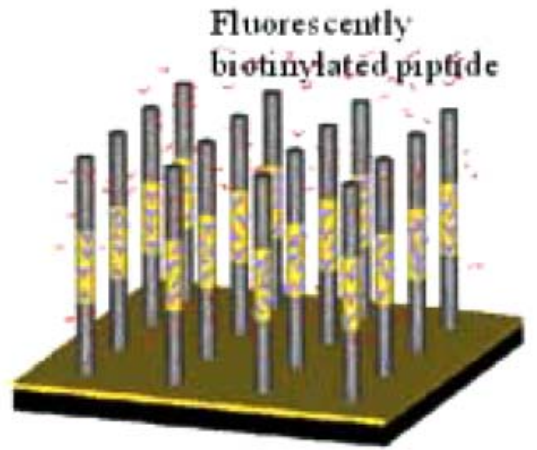

(d) was adhered on the silicon flake and dried (Fig. 3(a)). Then, the $\mathrm{AL}_{2} \mathrm{O}_{3}$ template was dissolved in $5 \mathrm{M} \mathrm{NaOH}$ at temperature of $40^{\circ} \mathrm{C}$ for $3 \mathrm{~h}$, followed by being dissolved in 0.5 $\mathrm{M} \mathrm{NaOH}$ for $0.5 \mathrm{~h}$ again. After this procedure, the $\mathrm{Al}_{2} \mathrm{O}_{3}$ was removed and the nanowires on the gold film were left on the silicon flake (Fig. 3(b)). Before being functionalized by fluorescently labeled ssDNA with thiol group and the biotinylated peptide, the nanowires adhered on the silicon flake should be immersed in double distilled water repratedly till the $\mathrm{pH}$ value is 7.0 to remove the residual $\mathrm{NaOH}$. Then, the gold segments of the nanowires were functionalized by thiol group modified ssDNA by incubation in a $5 \mu \mathrm{M}$ solution of fluorescently ssDNA with thiol group (Fig. 3(c)). After $30 \mathrm{~min}$, the silicon flake was immersed into buffer B several times to remove the free fluorescent ssDNA. Next, the silicon flake with nanowires was immersed into a $20 \mu \mathrm{M}$ solution of the biotinylated peptide labeled by the FITC to assemble the biotinylated peptide on the nickel segment of the nanowires (Fig. 3(d)). Then, the nanowires on the silicon were washed by the double distilled water and buffer B, respectively, to remove the free fluorescent peptide. Finally, the nanowires were processed by stirring, and resuspended in the buffer B.

\subsection{Constructing $\mathrm{F}_{1}$-ATPase motor on the substrate}

In order to observe the rotation of the $\mathrm{F}_{1}$-ATPase motor, several procedures should be carried out to functionalize the glass surface. Initially, the glass cell (bought from Coring Inc.) was cleaned in a piranha solution (a mixture with $3: 7 \mathrm{v} / \mathrm{v}$ of $30 \% \mathrm{H}_{2} \mathrm{O}_{2}$ and $98 \% \mathrm{H}_{2} \mathrm{SO}_{4}$ ) for $25 \mathrm{~min}$, and then rinsed in deionized (DI) water and dried in oven. Subsequently, $2 \%$ of APTES in $95 \%$ of acetone solution was infused into glass cell for two hours at normal temperature. After the surface was dried, the functionalized glass cell was immersed into $1.25 \%$ of glutaraldehyde solution for one hour at normal temperature. Then, the $\mathrm{Ni}_{\mathrm{AB}}-\mathrm{NTA}$ was added to the glass cell to functionalize the glass surface, followed by rinsing with acetone three times for $15 \mathrm{~min}$. Then, a $1 \mathrm{mM}$ solution of $\mathrm{Ni}_{2} \mathrm{SO}_{4}$ was infused into glass cell for half an hour, followed by washing away the free $\mathrm{Ni}_{2} \mathrm{SO}_{4}$. And the bovine serumalbumin, BSA, was coated for 8 hours at $4{ }^{\circ} \mathrm{C}$. Then, coupling $\mathrm{F}_{1}$-ATPase with the functionalized surface, we obtained the fixed engine for hybrid system. This glass cell was immersed in a $100 \mathrm{nM}$ solution of streptavidin for half an hour. Before every binding procedure, Buffer B $(10 \mathrm{mmol} / \mathrm{L}$ Hepes- $\mathrm{NaOH}$ set to $\mathrm{pH}$ $7.2,25 \mathrm{mmol} / \mathrm{L} \mathrm{KCl}$, and $5 \mathrm{mmol} / \mathrm{L} \mathrm{MgCl}_{2}$ ) should be used to wash away the residual material in the last process. After the suspensions with the functionalized nanowires were infused into the glass cell for several minutes, the glass cell was flushed by buffer B to remove the free nanowires. Then, the rotation of nanowire driven by $\mathrm{F}_{1}$-ATPase motors was observed by infused into a $2 \mathrm{mM}$ ATP solution, and recorded by using a charge-coupled device $(\mathrm{CCD})$ video camera. 


\section{Results and discussion}

Different nanowires were fabricated and functionalized to construct a hybrid device in this paper. Figure 2 shows the schematic approach of functionalizating the nickel nanowires and the corresponding products, in which Figures 2(a-2), (b-2) and (c-2) are the SEM picture of $\mathrm{Al}_{2} \mathrm{O}_{3}$ template, the TEM picture of the nickel nanowires and the video picture of fluorescently labeled nickel nanowires, respectively. From Fig. 2(a-2) and (b-2), the average diameter of nickel nanowires is $a=15 \pm 0.8 \mathrm{~nm}$. And the length of the nanowires can be controlled within a range of 1-2 microns by the deposition time (Fig. 2(c-2)). After being functionalized by the fluorescently labeled peptide, the fluorescently labeled nickel nanowires tethered to $F_{1}$-ATPase motors fixed on the cover glass by biotin-streptavidin-biotin linkage. The observations of nanowires were obtained when the buffer B dissolving ATP was infused into the liquid cell. Figure 4 shows the video microscopy image sequence of nickel nanowires driven by $\mathrm{F}_{1}$-ATPase motor. From the figure, some $120^{\circ}$ steps including occasional back steps were also observed, which are the same as Noji's results (Noji et al., 1997). It demonstrates that the nickel nanowires fabricated by the electrochemical deposition can be used to construct the hybrid device. It can be seen from the video of rotary nickel nanowires driven by $\mathrm{F}_{1}$-ATPase that $\mathrm{F}_{1}$-ATPase motor was attached to a random position along the nanowires. However, the construction of the hybrid device requires a controlling arrangement of biomoleculre on the required position on the nanowires. Multi segments along the nanowires can provide a variety of chemical functionalities, and can be used to control the arrangement of different molecules on it. Here, the multi-component nanowires, $\mathrm{Ni} / \mathrm{Au} / \mathrm{Ni}$, were fabricated. The gold segments of nanowires were functionalized by the fluorescent ssDNA based on the tightly binding between thiol group on ssDNA and the gold, and the nickel segment of nanowires was functionalized by fluorescently biotinylated peptide. The functionalization is shown as in Fig. 3. By using this approach, the centrifugation was not used in the functionalization to minimize the aggregation of the nanowires. Figures 5(a) and 5(b) show the schematic system used for the observation of the motor device and the TEM pictures of the multi-component nanowires, respectively. By selectively functionalizing the specific segments in multicomponent nanowires, one may direct bind $\mathrm{F}_{1}$-ATPase motor to the nickel segment of nanowires by biotin-streptavidin linkage. Thus, the attached region can be determined. The rotations of the multi segment nanowires driven by $F_{1}$-ATPase were observed when $2 \mathrm{mM}$ ATP was infused in to the glass cell, and the sequential images of one rotating motor are shown in Fig. 6. Figures 7 and 8 show the cumulated angle versus the different length of the propellers and the rotational speed in revolutions per second versus the length of the nanowires, respectively. The highest rotational speed of the nanowires observed in our experiments was 3.7 r.p.s. for the $0.7 \mu \mathrm{m}$ nanowire. The average frequency of the rotation of nanowires was lower than that of the fluorescently labeled actin filement driven by $\mathrm{F}_{1}$-ATPase motor (Noji et al., 1997) and that of propeller made of PMMA by Montemagno (Soong et al., 2000; Montemagno and Bachand, 1999). The total drag torque is calculated as

$$
\tau=\frac{4 \pi \eta L^{3} \omega}{3[\ln (L / 2 r)-0.447]},
$$

where $\xi=(4 \pi / 3) \eta L^{3} /[\ln (L / 2 r)-0.447]$ is the rotational frictional drag coefficient, $L$ the length of the nanowire, $r$ the radius of the nanowire, $\eta$ the viscosity of the medium, and $\omega$ the angular velocity. The average drag torque calculated by Eq. (1) was about $20 \mathrm{pN} \cdot \mathrm{nm}$ as shown in Fig. 8. The experimental result is within the range of $20-40 \mathrm{pN} \cdot \mathrm{nm}$, which is consistent with the results of previous reports (Noji et al., 1997; Soong et al., 2000; Yasuda et al., 1998; Montemagno and Bachand, 1999). The energy for one revolution of the propeller can be calculated as

$E=2 \pi \cdot \tau$
Fig. 4 Sequential images showing a rotating nickel nanowire driven $\mathrm{F}_{1}$-ATPase motor at $2 \mathrm{mM}$. Length from axis to tip is $1.8 \mu \mathrm{m}$, rotary rate is 0.7 r.p.s., and time interval between images is $40 \mathrm{~ms}$

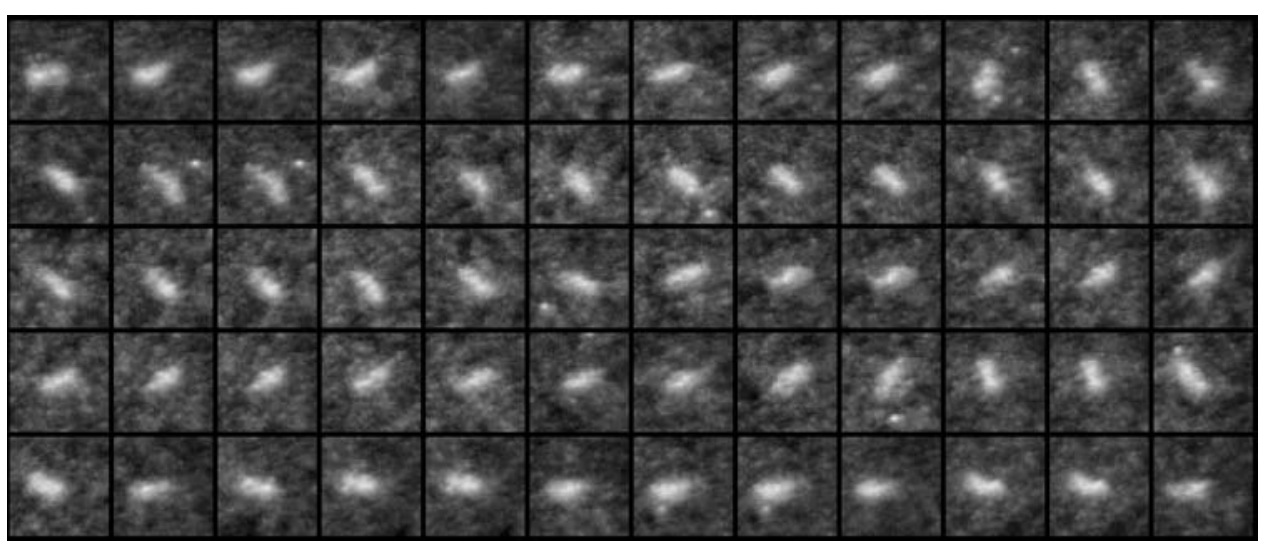


Fig. 5 Schematic of rotation of multi-component nanowire driven by $\mathrm{F}_{1}$-ATPase motor. (a) The nickel segment on nanowire was used as the region attached to the rotating shaft on $\mathrm{F}_{1}$-ATPase motor, (b) TEM picture of three segments nanowires

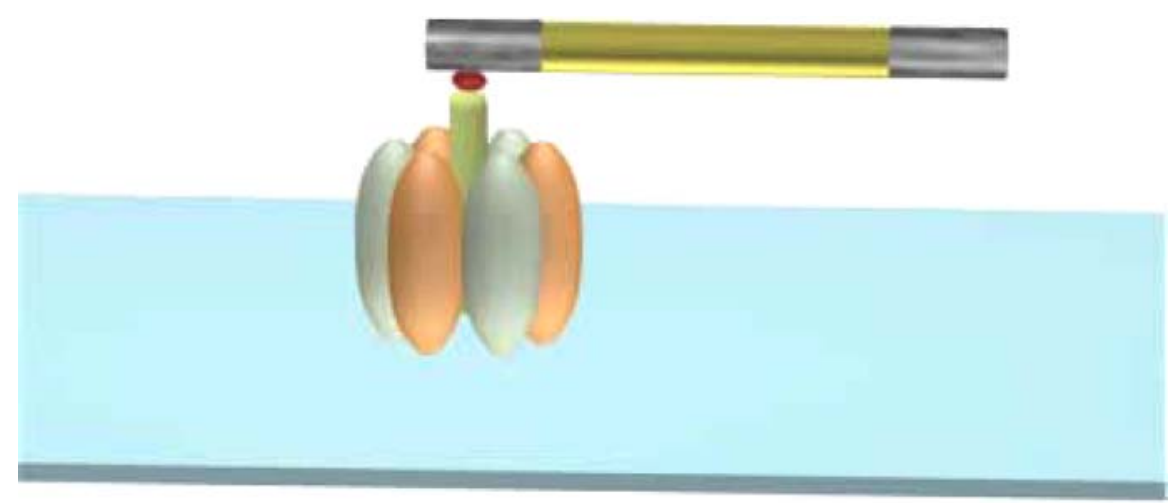

(a)

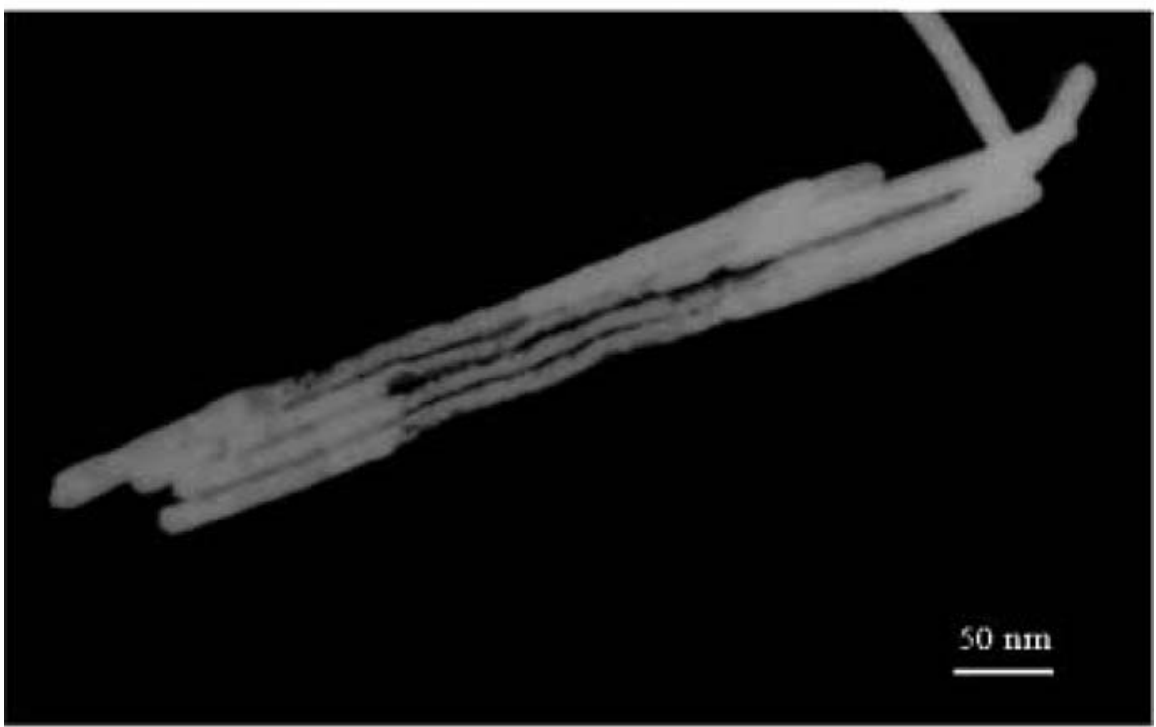

(b)

This means that the energy required to complete one revolution of the propellers in our experiment is about $125 \mathrm{pN} \cdot \mathrm{nm}$. However, about $240 \mathrm{pN} \cdot \mathrm{nm}$ of energy is released if three ATP molecules are hydrolyzed in accordance to physiological conditions (Stryer, 1995). Thus, the efficiency of motor is estimated as $50 \%$, which is consistent with the previous result (Soong et al., 2000). He thinks that the free energy of ATP hydrolysis depends on the concentration of $\mathrm{Mg}^{2+}$ and $\mathrm{Ca}^{2+}$ (Soong et al., 2000; Montemagno and Bachand, 1999), and is within a range of $90-150 \mathrm{pN} \cdot \mathrm{nm}$ (Yasuda et al., 1998; Montemagno and Bachand, 1999), which means that the efficiency in our experiment is not $100 \%$. Another reasons as given by Noji is that the obstructions or impeding factors such as the higher-than-bulk friction near the glass surface would reduce the rate, a precise estimation can not be obtained under five revolutions of motors (Noji et al., 1997; Yasuda et al., 1998). However, our experiments demonstrates that the multi-component nanowires can be used as propellers

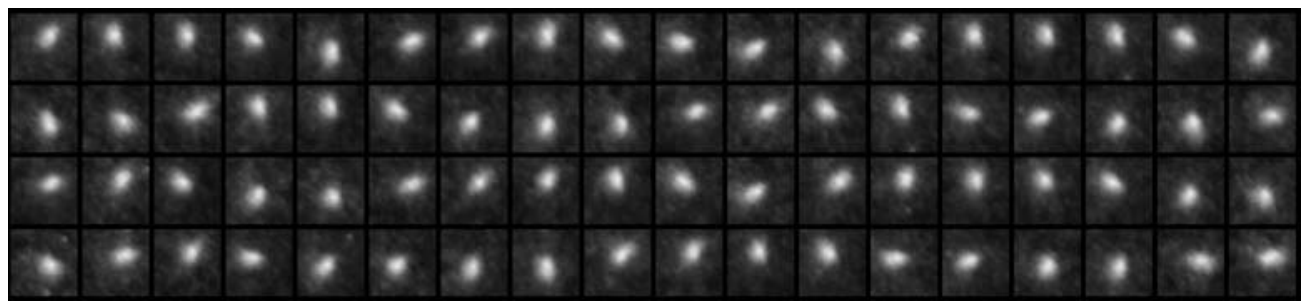

Fig. 6 Sequential images showing a rotating three segments nanowire driven by $\mathrm{F}_{1}$-ATPase motor at $2 \mathrm{mM}$, in which $\mathrm{F}_{1}$-ATPase motor attaches to the nickel segment of the multi-component nanowire. Length from axis to tip is $0.7 \mu \mathrm{m}$, rotary rate is 3.7 r.p.s., and time interval between images is $40 \mathrm{~ms}$ 


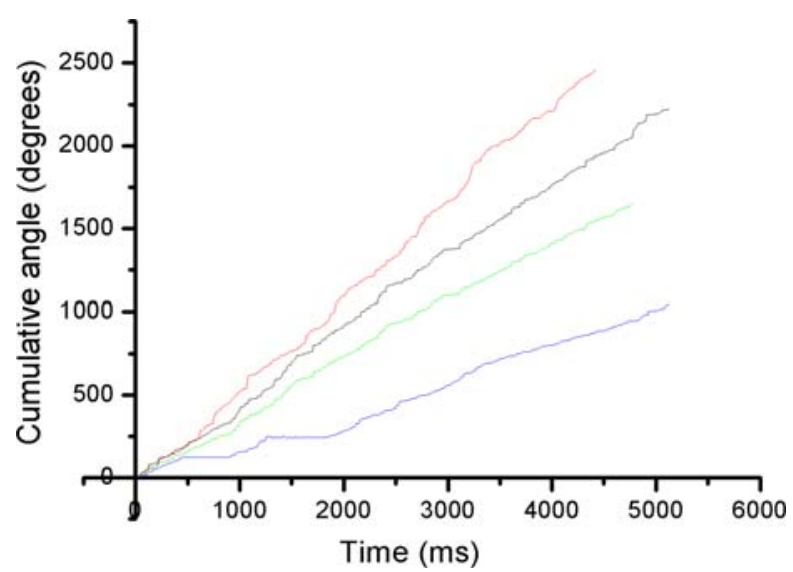

Fig. 7 Time course of $F_{1}$-ATPase rotation. Each line represents data from a rotating nanowire

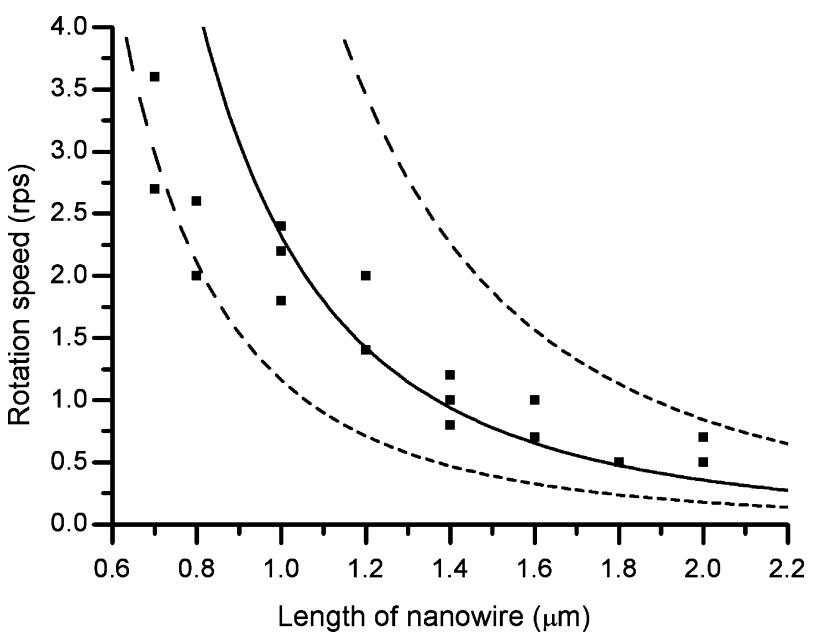

Fig. 8 Rotational speed in revolutions per second vs the length of the nanowires. Lines show the rotational speed under a constant torque of $20 \mathrm{pN} \cdot \mathrm{nm}$ (solid line), $40 \mathrm{pN} \cdot \mathrm{nm}$ (upper dotted line) and $10 \mathrm{pN} \cdot \mathrm{nm}$ (lower dotted line)

of motors, and provide a preferential attached region for the $\mathrm{F}_{1}$-ATPase motor.

\section{Conclusions}

To summarize, nickel nanowires were fabricated by electrochemical deposition in nanoporous templates. After being functionalized by fluorescently biotinylated peptide, the nickel nanowires can be assembled onto the $\mathrm{F}_{1}$-ATPase motor which is fixed on solid support as a hybrid device. In addition, multi-component nanowires, including gold and nickel, were designed and synthesized by electrochemical deposition into alumina filter templates. With a sequence of procedures, the gold segment and the nickel segment on the multicomponent nanowires were functionalized selectively by the thiol group modified ssDNA and the biotinylated peptide, respectively. Therefore, the biotinylated $\mathrm{F}_{1}$-ATPase motors can be attached to a desired position on the multi-component nanowires by biotin-streptavidin linkage. The procedures of functionalizing the nanowires do not involve the centrifugation, so the aggregation of the nanowires might be avoided at most. The successfully assembled approach adds the complexity of the hybrid device, but makes it possible to control the arrangement of the different biomolecules on the designed nanoscale structures. This approach will provide a variety of applications from patterning of proteins and cells to the physical positioning of nanowires.

\section{Acknowledgments}

This research was supported by Distinguished Young Scholar Fund of National Natural Science Foundation of China (NSFC, Grant No. 10225209), key project from Chinese Academy of Sciences (Grant No. KJCX-SW-L2) and NSFC project (Grant No. 90305020).

\section{References}

J.P. Abrahams, A.G.W. Leslie, R. Lutter, and J.E. Walker, Nature 370, 621-628 (1994).

G.D. Bachand and C.D. Montemagno, Biomedical Microdevices 2, 179-184 (2000).

R. Berry and J. Armitage, Advances in Microbial Physiology 41, 291337 (1999).

S.M. Block, Cell 93, 5-8 (1998).

P.D. Boyer, Biochim. Biophys. Acta 1140, 215-250 (1993).

G.B. Cox, A.L. Fimmel, F. Gibson, and L. Hatch, Biochim. Biophys. Acta 849, 62-69 (1986).

I. Dobbie, M. Linari, G. Piazzesi, M. Reconditi, N. Koubassova, M. A. Ferenczi, V. Lombardi, and M. Irving, Nature 396, 383-387 (1998).

T.M. Duncan, V.V. Bulygin, Y. Zhou, M.L. Hutcheon, and R.L. Cross, Proc. Natl. Acad. Sci. 92, 10964-10968 (1995).

S. Engelbrecht and W. Junge, FEBS Lett. 414, 485-491 (1997).

J. Fritz, M.K. Baller, H.P. Lang, H. Rothuizen, P. Vettiger, E. Meyer, H.J. Güntherodt, C. Gerber, and J.K. Gimzewski, Science 288, 316-318 (2000).

I.R. Gibbons and J. Biol. Chem. 263, 15837-15840 (1988).

G. Groth and J. Walker, FEBS Lett. 410, 117-123 (1997).

T.J. Huang, B. Brough, C.M. Ho, Y. Li, A.H. Flood, P.A. Bonvallet, H.R. Tseng, J.F. Stoddart, M. Baller, and S. Magonov, Appl. Phys. Lett. 85, 5391-5393 (2004).

A.J. Hunt, F. Gittes, and J. Howard, Biophysical Journal 67, 766-781 (1994).

L. Jia, S.G. Moorjani, T.N. Jackson, and W.O. Hancock, Biomedical Microdevices 6, 67-74 (2004).

D. Kaiser, Curr. Biol. 10, 777-780 (2000).

K. Kitamura, M. Tokunaga, A.H. Iwane, and T. Yanagida, Nature 397, 129-134 (1999).

C. Lapoinet, A. Hltgren, D.M. Silevitch, E.J. Felton, D.H. Reich, and R.L. Leheny, Science 303, 652-655 (2004).

B.S. Lee, S.C. Lee, and L.S. Holliday, Biomedical Microdevices 5, 269-280 (2003).

T. Matsui and M. Yoshida, Biochimica et Biophysica ACTABioenergetics 1231, 139-146 (1995). 
C.D. Montemagno and G.D. Bachand, Nanotechnology 10, 225-231 (1999).

K. Nishio, A. Iwamoto-Kihara, A. Yamamoto, Y. Wada, and M. Futai, Proc. Natl. Acad. Sci. 99, 13448-13452 (2002).

H. Noji, R. Yasuda, M. Yoshida, and K. Jr. Kinosita, Nature 386, 299 302 (1997).

I. Ogilvie, R. Aggeler, and R.A. Capaldi, J. Biol. Chem. 272, 16652 16656 (1997).

H. Omote, N. Sambonmatsu, K. Saito, Y. Sambongi, A.I. Kihara, T. Yanagida, Y. Wada, and M. Futai, Proc. Natl. Acad. Sci. 96, 77807784 (1999).

G. Oster and H. Wang, Trends Cell Biol. 13, 114-121 (2003).

O. Pänke, K. Gumbiowski, W Junge, and S. Engelbrecht, FEBS Lett. 472, 34-38 (2000).

D.H. Reich, M. Tanase, A. Hultren, L.A. Bauer, C.S. Chen, and G.J. Meyer, J. Appl. Phys. 93, 7275-7280 (2003).
Q. Ren, Y. P. Zhao, L. Han, and H. B. Zhao, Nanotechnology 17, 17781785 (2006).

A.K. Salem, J. Chao, K.W. Leong, and P.C. Searson, Advanced Materials 16, 268-271 (2004).

C. Shingyoji, H. Higuchi, M. Yoshimura, E. Katayama, and T. Yanagida, Nature 393, 711-714 (1998).

R.K. Soong, G.D. Bachand, H.P. Neves, A.G. Olkhovets, H.G. Craighead, and C.D. Montemagno, Science 290, 1555-1558 (2000).

R.K. Soong, H.P. Neves, J.J. Schmidt, G.D. Bachand, and C.D. Montemagno, Biomedical Microdevices 3, 71-73 (2001).

L. Stryer, Biochemistry (Freeman, New York, 1995) p. 443.

R. Yasuda, H. Noji, K. Jr. Kinosita, and M. Yoshida, Cell 93, 1117-1124 (1998).

R. Yasuda, H. Noji, M. Yoshida, K. Jr. Kinosita, and H. Itoh, Nature 410, 898-904 (2001). 